Bull. Mater. Sci., Vol. 35, No. 1, February 2012, pp. 7-12. (c) Indian Academy of Sciences.

\title{
Recent analytical applications of nanoparticle sensitized lucigenin and luminol chemiluminescent reactions
}

\author{
S M WABAIDUR*, MU NAUSHAD and $Z$ A ALOTHMAN \\ Department of Chemistry, College of Science, Building-5, King Saud University, Riyadh, Saudi Arabia
}

MS received 16 June 2010; revised 26 March 2011

\begin{abstract}
There is an ever-increasing demand for rapid, sensitive, cost effective and selective detection methods for the analysis of many essential compounds. When chemiluminescence has been introduced to analytical chemistry as a detection technique, it has been shown to meet many of these requirements. This method has become a powerful tool for the determination of many compounds. Using this method, low detection limits can be obtained with simple and inexpensive instrumentation. Coupled with flow injection technique the method has become more popular for wider applications. Since many excellent reviews on the chemiluminogenic techniques have appeared in the literature in recent years, the present paper does not intend to cover the exhaustive studies in this area, but will selectively describe the analytical applications of nanoparticle sensitized lucigenin and luminol chemiluminescent reactions and evaluate their recent progress together with our present work.
\end{abstract}

Keywords. Chemiluminescence; flow injection analysis; lucigenin; detection methods.

\section{Introduction}

An emission of electromagnetic radiation (ultraviolet, visible, or infrared) produced by molecules that are electronically excited during the chemical reaction is known as chemiluminescence (CL). The decay of the excited state to a lower energy level is responsible for the emission of light. As the emission of light is a function of the concentration of the chemical species involved in the chemiluminescence reaction, measurement of emission intensities can be used for analytical purposes. Chemiluminescence differs from fluorescence in that the electronic excited state is derived from the product of a chemical reaction rather than the more typical way of creating electronic excited states, viz. absorption. It is the antithesis of a photochemical reaction, in which light is used to drive an endothermic chemical reaction. Here, light is generated from a chemically exothermic reaction. A standard example of chemiluminescence in the laboratory setting is the luminol test. Here, blood is indicated by luminescence upon contact with iron in hemoglobin. When chemiluminescence takes place in living organisms, the phenomenon is called bioluminescence. A light stick emits light by chemiluminescence. Chemiluminescent techniques can be applied in two types of analytical methods: direct method and indirect method. In direct method, a substrate and an oxidant in the presence of some cofactors and catalyst react to yield a product or intermediate. A fraction of the product or intermediate is formed in an electronically excited state and

\footnotetext{
*Author for correspondence (tarabai22@yahoo.com.sg)
}

subsequently goes to the ground state with emission of light and in indirect method, this process involves transfer of energy of the excited species to a fluorophore like terbium sensitized chemiluminescence and post chemiluminescence.

The most popular chemiluminescent systems include luminol and lucigenin. The important features of chemiluminescent systems are good sensitivity, simple instrumentation, low detection limit, wide range of applications and large linear range of analytes. Because of these advantages, chemiluminescence occupies a position as one of the more sensitive techniques of trace analysis of essential compounds. Alves et al (2003) established a luminal and a lucigenin dependent chemiluminescence methods to investigate the role of receptors for $F c$ portion of $I g G(F c \gamma R)$ and/or complement receptors $(\mathrm{CR})$ in mediating the oxidative burst in neutrophils from systemic lupus erythematosus (SLE) patients compared with healthy controls. In the luminal-CL system, all the reactive oxygen species (ROS) are responsible for light production, whereas in the lucigenin-CL system, only the first ROS generated, converts the lucigenin into an unstable intermediate molecule, which also emits light. First, neutrophils from healthy controls and SLE patients were stimulated with different IC opsonized or not with complement from normal human serum (NHS) or SLE serum, in presence of $10^{-4} \mathrm{M}$ luminol. To improve the sensitivity of chemiluminescence detection, metal catalyzed chemiluminescence has been extensively applied to bioanalysis and immunoassays. Most recently nanoparticles are frequently used as they display unusual catalytic properties for a number of chemical reactions, depending upon their size, shape and stabilizing agents. However, the applicability of nanoparticles as catalysts in chemiluminescence reaction is 
still unknown. It was found that lucigenin alkaline solution could react with hydrazine in the presence of platinum nanoparticles to generate strong CL centred at $480 \mathrm{~nm}$. It is suggested that the CL enhancement might be due to the fact that thiol-containing compounds could facilitate the electron transfer process under the catalysis of Pt nanoparticles and accelerate the generation of $\mathrm{OH}$, and $\mathrm{O}_{2}^{-}$radicals, leading to the strong CL (Liu et al 2011). A CL system was developed for the detection of fluorescent organic compounds (FOCs). The CL system is composed of an oxidizing agent, peroxomonosulfate $\left(\mathrm{KHSO}_{5}\right)$ and a metal ion catalyst, cobalt (as sulfate). In unbuffered conditions, $\mathrm{HSO}_{5}^{-}$is decomposed by $\mathrm{Co}^{2+}$ to yield reactive species $\left(\mathrm{Co}^{3+}, \mathrm{SO}_{4}^{-}\right.$, etc), which are strong oxidizing agents leading to CL from FOCs (Tsukada et al 1998). Chen et al (2007) found that silver nanoparticles (AgNPs) could enhance the CL intensity of luminol$\mathrm{H}_{2} \mathrm{O}_{2}$ system. In this reaction, luminol intermediate is generated under alkaline condition on the surface of AgNPs in luminol- $\mathrm{H}_{2} \mathrm{O}_{2}$ system and enhances $\mathrm{CL}$ intensity.

Since a number of reviews on the chemiluminescence techniques in analytical chemistry have appeared in recent years, the present paper does not intend to cover the exhaustive research on this area, but selectively describe key references and recent advances together with our current work. Recent trends will be focused in the last section on the use of nanoparticles for significant improvements in CL detection systems.

\section{Analytical applications}

Chemiluminescence (CL) analysis has been frequently used for the analysis of organic and inorganic species because of its low detection limit, wide dynamic range and simple instrumentation. Recently, Du et al (2007) used lucigenin CL reaction with flow injection technique for the determination of $\mathrm{Cr}$ (III) and total Cr. Strong chemiluminescence signal was produced from the reaction of $\mathrm{Cr}(\mathrm{III})$, lucigenin and $\mathrm{KIO}_{4}$ in alkaline media. The determination of total $\mathrm{Cr}$ was carried out by prereduction of $\mathrm{Cr}(\mathrm{VI})$ to $\mathrm{Cr}(\mathrm{III})$ by using $\mathrm{H}_{2} \mathrm{SO}_{3}$. The detection was reported here $\left(1 \times 10^{-10} \mathrm{~g} / \mathrm{ml}\right)$. To test the applicability of the method water sample was analysed for $\mathrm{Cr}$ (III) and total $\mathrm{Cr}$.

Determination of epinephrine using electrochemiluminescence (ECL) of lucigenin system by the anodic potential sweep had been performed (Su et al 2005). ECL response of lucigenin was found at anodic potential above $1.1 \mathrm{~V}$ (vs $\mathrm{Ag} / \mathrm{AgCl}$ ) in non-ionic surfactant Triton $\mathrm{X}-100$ and the ECL signal was significantly enhanced by epinephrine. Under optimum condition, enhanced ECL intensity was linear with the epinephrine concentration in the range of $4.0 \times$ $10^{-8}$ to $2.0 \times 10^{-7} \mathrm{M}$ and the detection limit (defined as $S / N=3$ ) was found to be $2.4 \times 10^{-8} \mathrm{M}$. The method was successfully applied to the determination of epinephrine in pharmaceutical samples with satisfactory results. The reaction mechanism for the lucigenin ECL system in the presence of epinephrine was also discussed.
$\mathrm{Li}$ and Lee (2005) developed a new chemiluminescence (CL) method for the selective determination of As(III) and $\mathrm{As}(\mathrm{V})$ ions in aqueous solution using a FIA system. The method was based on the increased CL intensity with the addition of $\mathrm{As}(\mathrm{V})$ ion into a solution of lucigenin and hydrogen peroxide. The addition of As(III) ion into the solution did not change CL intensity. Total concentration of As ions was determined after pre-oxidation of $\mathrm{As}(\mathrm{III})$ to $\mathrm{As}(\mathrm{V})$ with hydrogen peroxide in basic solution. The As(III) content was estimated by subtracting the content of $\mathrm{As}(\mathrm{V})$ ion from total As concentration. The effects of concentrations of $\mathrm{KOH}$ and $\mathrm{H}_{2} \mathrm{O}_{2}$, and flow rates of reagents on CL intensity had been investigated. The calibration curve for $\mathrm{As}(\mathrm{V})$ ion was linear over a range from $1.0 \times 10^{-2}$ to $10 \mu \mathrm{g} / \mathrm{g}$, the coefficient of correlation was 0.997 and the detection limit was $5.0 \times 10^{-3} \mu \mathrm{g} / \mathrm{g}$ under the optimal experimental conditions. Lucigenin has been used to determine catechin in aqueous solution by measuring chemiluminescence intensities using a stopped flow system (Lee et al 2005). The lucigeninhydrogen peroxide chemiluminescence reaction was chosen for the determination of catechin. $\mathrm{Fe}$ (II) ion was added to the chemiluminescence system to enhance the CL sensitivity. The chemiluminescence intensity from the lucigenin system was increased by the addition of catechin. Effects of flow rates of reagent and sample and concentrations of lucigenin, hydrogen peroxide, $\mathrm{Fe}(\mathrm{II})$ ion and $\mathrm{KOH}$ were studied. The calibration curve for catechin was linear over a range from $1.0 \times 10^{-6}$ to $1.0 \times 10^{-3} \mathrm{M}$ and the detection limit was $3.0 \times 10^{-7} \mathrm{M}$ under optimal experimental conditions.

Our group developed CL method combined with the flow injection analysis (FIA) technique (Lee et al 2007) for the quantification of bilirubin based on the quenching effect of bilirubin on CL from the lucigenin-hydrogen pe-



Figure 1. CL kinetic curves of system (I), lucigenin- $\mathrm{H}_{2} \mathrm{O}_{2}$ in absence of bilirubin, and system (II), lucigenin- $\mathrm{H}_{2} \mathrm{O}_{2}$ in presence of bilirubin (Conditions: lucigenin, $5 \times 10^{-4} \mathrm{M}$; bilirubin, $58.47 \mu \mathrm{g} / \mathrm{ml} ; \mathrm{H}_{2} \mathrm{O}_{2}, 2.5 \mathrm{M} ; \mathrm{NaOH}, 5 \mathrm{M}$; flow rate, $3.0 \mathrm{ml} / \mathrm{min}$; sample volume, $200 \mu \mathrm{l}$ (adapted from Chen et al (2011)). 


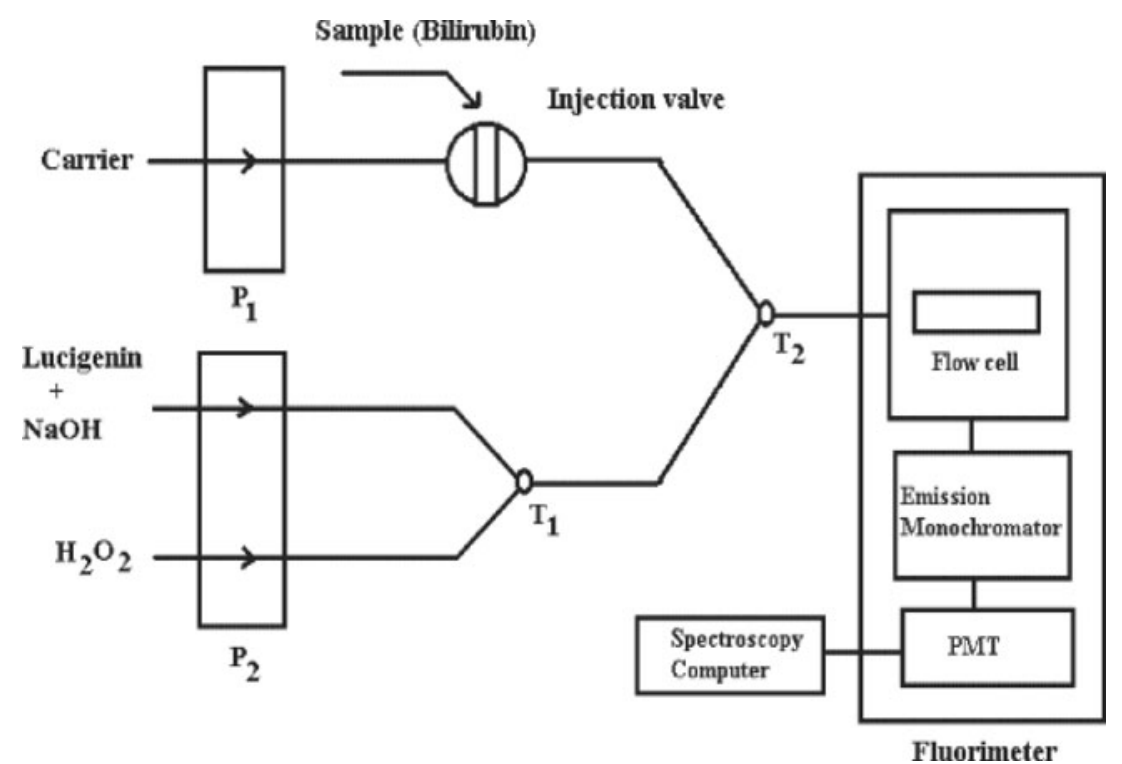

Figure 2. Schematic diagram of FIA-CL manifold employed for quantitative determination of bilirubin. $P_{1}, P_{2}$, peristaltic pumps; $T_{1}, T_{2}, Y$-pieces (adapted from Chen et al (2011)).

roxide system in an alkaline medium (figure 1). The flowinjection analysis (FIA) set up used in this study is shown in figure 2. Under the optimum conditions, decreased CL intensity was proportional to the concentration of bilirubin, in the range $0.0585-58.47 \mu \mathrm{g} / \mathrm{ml}$. The detection limit estimated from the calibration graph was about $7.8826 \mu \mathrm{g} / \mathrm{ml}$. The relative standard deviation (RSD) of 10 parallel measurements $\left(1 \times 10^{-4} \mathrm{M}\right.$ bilirubin) was $2.5 \%$. Recoveries of bilirubin were found to fall in the range $94-97.5 \%$ using control sera. The method is interference-free, fast and easy to carry out.

Concerning the reaction mechanism, the $\mathrm{CL}$ inhibition by bilirubin of the lucigenin- $\mathrm{H}_{2} \mathrm{O}_{2}$ reaction was proposed to be due to the competition with lucigenin for hydrogen peroxide. Bilirubin reacted with oxidant $\mathrm{H}_{2} \mathrm{O}_{2}$ to partly consume $\mathrm{H}_{2} \mathrm{O}_{2}$, forming biliverdin. Thus, bilirubin inhibited the CL intensity of the lucigenin- $\mathrm{H}_{2} \mathrm{O}_{2}-\mathrm{NaOH}$ system. This phenomenon was confirmed by a preliminary UV-vis spectroscopic study. From the study it was observed that bilirubin had a maximum absorption peak at $434 \mathrm{~nm}$. Addition of $\mathrm{H}_{2} \mathrm{O}_{2}$ to bilirubin resulted in a gradual change of the bilirubin yellow colour to the green biliverdin, and the biliverdin peak appeared at $291 \mathrm{~nm}$ simultaneously with the diminution of the bilirubin peak at $434 \mathrm{~nm}$.

A simple continuous-flow CL system was reported by Li et al (2007), for simultaneous determination of glucose, fructose and lactose in ternary mixtures of reducing sugars without previous separation. The method was based on the different kinetics of the individual sugars in the oxidation reaction with potassium ferricyanide. The known luminol$\mathrm{K}_{3} \mathrm{Fe}(\mathrm{CN})_{6} \mathrm{CL}$ system was used to measure the kinetic data of the system. The CL intensity was measured and recorded every second from 1 to $300 \mathrm{~s}$. The data obtained were processed chemometrically using an artificial neural network. The relative standard errors of prediction for three analytes were $<5 \%$. The proposed method was successfully applied to the simultaneous determination of three sugars in some food samples.

A flow-injection procedure was reported for the determination of carbaryl based on its inhibition effect on the chemiluminescence from luminol-cobalt(II) system in alkaline medium in the presence of hydrogen peroxide (Waseem 2007). The calibration curve was found over a range $5.0 \times$ $10^{-7}$ to $20 \times 10^{-6} \mathrm{M}$ and the detection was $2.37 \times 10^{-7} \mathrm{M}$ for carbaryl. The effects of some carbamates, anions, and cations were investigated on luminol CL system for the determination of carbaryl. The proposed method was applied for the assay of carbaryl in natural waters.

Li et al (2007) reported a flow injection chemiluminescence method for the determination of four phenothiazine drugs, such as chlorpromazine hydrochloride, perphenazine hydrochloride, fluphenazine hydrochloride and thioridazine hydrochloride. Intense CL signal was produced when the drug was injected into the mixed stream of luminol with $\mathrm{KMnO}_{4}$. The dynamic ranges of the method were $0.0020-1.0 \mu \mathrm{g} / \mathrm{ml}$ chlorpromazine hydrochloride, 0.0040 $3.0 \mu \mathrm{g} / \mathrm{ml}$ perphenazine hydrochloride, $0.0020-5.0 \mu \mathrm{g} / \mathrm{ml}$ fluphenazine hydrochloride and $0.0050-1.0 \mu \mathrm{g} / \mathrm{ml}$ thioridazine hydrochloride. The detection limits were found to be $0.4 \mathrm{ng} / \mathrm{ml}$ chlorpromazine hydrochloride, $0.7 \mu \mathrm{g} / \mathrm{ml}$ perphenazine hydrochloride, $2 \mu \mathrm{g} / \mathrm{ml}$ fluphenazine hydrochloride and $0.7 \mu \mathrm{g} / \mathrm{ml}$ thioridazine hydrochloride.

Picogram-level gatifloxacin was determined by Xie et al (2007) which was based on its significantly catalytic effect on myoglobin-luminol CL reaction in FIA system. The 
enhanced chemiluminescence intensity was proportional to the concentration of gatifloxacin in the range from $50 \mu \mathrm{g} / \mathrm{L}-10 \mu \mathrm{g} / \mathrm{L}$, and the detection limit was $20 \mathrm{mg} / \mathrm{L}$. The method was applied to the determination of gatifloxacin in tablets, human serum and urine samples with the recovery from 97.4-104.5\%. DNAzyme-functionalized Au-NPs act as catalytic labels for the amplified detection of DNA and telomerase activity on nucleic acid-functionalized gold surface. The DNAzyme stimulates, in the presence of hemin, $\mathrm{H}_{2} \mathrm{O}_{2}$, and luminol, the generation of chemiluminescence (Niazov et al 2004). It was found that $\mathrm{Au}-\mathrm{Ag}$ alloy nanoparticles could enhance the chemiluminescence of rhodamine $6 \mathrm{G}$-cerium(IV) system, and the most intensive CL signals were obtained by $\mathrm{Au}-\mathrm{Ag}$ alloy nanoparticles with a molar ratio of 3:4 (Li et al 2009a, b, c). Sau et al (2001) addressed the gold particle catalyzed eosin reduction in the presence of $\mathrm{NaBH}_{4}$. The catalytic activity of the gold nanoparticles is different for different size regimes (size domain dependent) of the particles. It has been shown that the kinetics of the catalytic rate has a bearing with the particle size (viz. surface area, mass, etc). The kinetics of catalytic reduction revealed that for the same surface area of different particles, the catalytic rate does not increase proportionately with the decrease in size over the whole range of average diameter from 10 to $46 \mathrm{~nm}$. The effect of $\mathrm{ZnO}$ nanoparticles on the luminol- $\mathrm{H}_{2} \mathrm{O}_{2} \mathrm{CL}$ system, a popular model CL system, was investigated. It was found that $\mathrm{ZnO}$ nanoparticles with a size regime from 6 to $21 \mathrm{~nm}$ could enhance CL of the luminol$\mathrm{H}_{2} \mathrm{O}_{2}$ system, and the strongest CL signal was obtained when the mean diameter of $\mathrm{ZnO}$ nanoparticles was $6 \mathrm{~nm}$ ( $\mathrm{Li}$ et al $2009 a-c)$. It was found that gold nanoparticles with different sizes could enhance the $\mathrm{CL}$ of the luminol- $\mathrm{NaIO}_{4}$ system in alkaline solution. The most intensive CL signals were obtained with gold nanoparticles having a diameter of $4 \mathrm{~nm}$ and CL intensity increased linearly with the concentration of gold nanoparticles ( $\mathrm{Li}$ et al 2008). It was found that gold nanoparticles of small size $(<5 \mathrm{~nm})$ could inhibit the $\mathrm{CL}$ of the luminol-ferricyanide system, whereas gold nanoparticles of large size $(>10 \mathrm{~nm})$ could enhance this CL, and the most intensive CL signals were obtained with $25 \mathrm{~nm}$ diameter gold nanoparticles (Guo and Cui 2007). The CL intensity of the luminol- $\mathrm{KIO}_{4}$ system is strongly enhanced by addition of zinc sulfide nanoparticles (ZnS-NPs) capped with 3-mercaptopropionic acid. On injection of fluoroquinolones into the luminol- $\mathrm{KIO}_{4}-\mathrm{ZnS}-\mathrm{NP}$ system, CL intensity is substantially increased. The CL emission peaks at $430 \mathrm{~nm}$, thus indicating that the luminophore is 3-aminophthalate (Li et al 2009a-c). It was found that noble metal nanoparticles including $\mathrm{Ag}, \mathrm{Au}$ and $\mathrm{Pt}$ nanoparticles in the presence of adsorbates such as iodide ion, cysteine, mercaptoacetic acid, mercaptopropionic acid, and thiourea could reduce lucigenin (bis- $N$-methylacridinium) to produce chemiluminescence (Xu and Cui 2007). Platinum colloids were found to enhance the CL of the luminol- $\mathrm{H}_{2} \mathrm{O}_{2}$ system, and the most intensive CL signals were obtained with citrate-protected Pt colloids synthesized with citrate as both a reductant and a stabilizer (Cui et al 2005). Zhang et al (2006) found that potassium permanganate $\left(\mathrm{KMnO}_{4}\right)$ could react with gold nanoparticles in a strong acid medium to generate particle size-dependent CL. For gold nanoparticles with a size of 2.6 or $6.0 \mathrm{~nm}$, the reaction was fast and could produce the excited state, $\mathrm{Mn}(\mathrm{II})$, with light emission around $640 \mathrm{~nm}$. For gold nanoparticles larger than $6 \mathrm{~nm}$, no light emission was observed due to a much slower reaction rate. The CL intensity was found to increase linearly with the concentration of $2.6 \mathrm{~nm}$ gold nanoparticles. The reaction of gold nanoparticles with a potassium periodate-sodium hydroxide-carbonate system undergoes chemiluminescence with three emission bands at 380-390, 430-450, and 490$500 \mathrm{~nm}$, respectively. It was found that the light intensity increased linearly with the concentration of gold nanoparticles, and CL intensity increased dramatically when the citrate ions on the nanoparticle surface were replaced by $\mathrm{SCN}^{-}$(Cui et al 2005). The mixtures of silver(I) and citrate that are used to produce silver nanoparticles evoke intense chemiluminescence with tris(2,2'-bipyridyl)ruthenium(II) and cerium(IV), which can be exploited for the determination of citrate ions and other analytes over a wide concentration range (Gorman et al 2007). Zhang et al (2002) observed chemiluminescence on introducing ethanol vapour to the surface of nanosized $\mathrm{ZrO}_{2}$ and this effect could be utilized to design a sensor for trace ethanol detection. Cui and Dong (2006) indicated that the strong ECL signals of lucigenin system could be obtained on a gold nanoparticle self-assembled gold electrode both in alkaline and neutral solutions, which is even stronger than that of luminol system on a gold nanoparticle self-assembled gold electrode under optimization conditions. Moreover, the gold nanoparticle self-assembled gold electrode also exhibits good stability for the lucigenin ECL system, overcoming the problem of electrode fouling on a traditional electrode. It was found that lucigenin alkaline solution could react with hydrazine in the presence of Pt nanoparticles to generate strong CL centred at $480 \mathrm{~nm}$. The bis-acridinium dication lucigenine was successfully used as a molecular assembler in the functionalization of metal nanoparticles to facilitate approach of the pesticide to the metal surface. From the surface-enhanced Raman scattering (SERS) spectra valuable information about the interaction mechanism between the pesticide and lucigenin can be deduced. In fact, endosulfan undergoes an isomerization upon adsorption onto the metal, while the viologen undergoes a rotation of the acridinium planes to better accommodate the pesticide molecule. An interaction between the $\mathrm{N}$ atom of the central acridinium ring and the pesticide $\mathrm{Cl}-\mathrm{C}=\mathrm{C}-\mathrm{Cl}$ fragment is verified through a charge-transfer complex. Sensitive detection of trace bisphenol A (BPA) in water samples has been accomplished via inhibition of luminol CL by BPA on the silver nanoparticles (AgNPs)-enhanced luminol- $\mathrm{KMnO}_{4} \mathrm{CL}$ system for the first time. Under the optimized experimental conditions, CL intensity was found to be proportional to the concentration of BPA ranging from $1.0 \times 10(-8)$ to $5.0 \times 10(-5) \operatorname{gL}(-1)$ (Chen et al 2011). The chemiluminescence immunoassay can be used for determining various analytes in samples. The probe and the assay can be applied 
to clinical diagnosis and treatment, pharmaceutical analysis, food safety inspection and environmental monitoring.

\section{Recent progress and conclusions}

A promising trend is to apply this sensitive chemistry in other techniques like liquid chromatography and capillary electrophoresis. One of the most sensitive and selective detection techniques for chromatography is chemiluminescence (CL), in which the emission of electromagnetic radiation (ultraviolet, visible or infrared) produced by a chemical reaction based on the decay of an excited species to the electronic ground-state is measured. A luminol chemiluminescence (CL) reaction was applied for the simultaneous determination of nine corticosteroids (triamcinolone, prednisolone, prednisone, cortisone, betamethasone, dexamethasone, corticosterone, flumethasone and triamcinolone acetonide) using flow injection. The luminol solution was made in $\mathrm{NaOH}$ with hexacyanoferrate(II). Hexacyanoferrate(III) was used as an oxidant. Adequate isocratic chromatographic separation was obtained using a simple mobile phase (acetonitrile-water). The kinetic aspects of chemiluminescence reactions are very important in the sense that maximum intensity occurs simultaneous to the arrival of the substance in the detection cell. Under the optimal experimental conditions, the lowest range injected was $15 \mathrm{mg} / \mathrm{l}$ for prednisolone, prednisone and cortisone, and the highest was $2 \mathrm{mg} / \mathrm{l}$ for betamethasone. Reproducibility and repeatability ranged from 2.59 to 7.59 and 1.50 to 3.61 for different corticosteroids, respectively.

Capillary electrophoresis (CE) is an advanced analytical technique. It has advantages of simplicity, high efficiency, low cost and short analysis time. CE with electrochemical detection or UV detection has already been applied for determination of a number of compounds but the major drawback of CE with UV detection is its sensitivity. As chemiluminescence (CL) detection is one of the most sensitive detection technologies and the costs of the instrumental set up are relatively low, CL detection has become an attractive detection tool for sensitive detection in capillary electrophoresis. Zhao et al (2007) reported a rapid and simple method using capillary electrophoresis (CE) with CL detection for the determination of levodopa. This method was based on enhancement effect of levodopa on the CL reaction from luminol and potassium hexacyanoferrate(III) $\left(\mathrm{K}_{3}\left[\mathrm{Fe}(\mathrm{CN})_{6}\right]\right)$ in alkaline aqueous media. The optimized conditions for the CL detection were $1.0 \times 10^{-5} \mathrm{M}$ luminol added to the $\mathrm{CE}$ running buffer and $5.0 \times 10^{-5} \mathrm{M}$ $\mathrm{K}_{3}\left[\mathrm{Fe}(\mathrm{CN})_{6}\right]$ in $0.6 \mathrm{M} \mathrm{NaOH}$ solution introduced post column. Under optimized conditions, the linear range from $5.0 \times 10^{-8}$ to $2.5 \times 10^{-6} \mathrm{M}$, and the detection limit of $2.0 \times 10^{-8} \mathrm{M}$ for levodopa were obtained.

Recently, metal nanoparticles have been investigated as effective catalysts for the chemiluminescence reaction. Zhang et al (2005) observed that gold nanoparticles with a size regime from 6 to $99 \mathrm{~nm}$ could enhance CL from the luminol- $\mathrm{H}_{2} \mathrm{O}_{2}$ system and the chemiluminescence enhancement by gold nanoparticles of the system was supposed to produce from the catalysis of gold nanoparticles, which facilitated the radical generation and electron-transfer processes taking place on the surface of the gold nanoparticles.

It was reported that gold nanoparticles with different sizes enhance the CL of the luminol- $\mathrm{NaIO}_{4}$ system in alkaline solution. Gold nanoparticles having a diameter of $4 \mathrm{~nm}$ give most intensive CL signals ( $\mathrm{Li}$ et al 2008) and CL intensity increased linearly with the concentration of gold nanoparticles. Catechol, hydroquinone and resorcinol were found to quench the chemiluminescence intensity from the luminol$\mathrm{NaIO}_{4}$ reaction catalyzed by gold nanoparticles, which made it applicable for the determination of these polyphenols. Under the optimum experimental conditions, the detection limits were in the range of $2.1 \times 10^{-9} \mathrm{~g} / \mathrm{ml}$ to $1.0 \times$ $10^{-10} \mathrm{~g} / \mathrm{ml}$. The method was successfully applied to the determination of catechol in tap water and synthetic samples.

In conclusion, nanoparticle sensitized chemiluminescence has a promising future in analytical chemistry. Versatility of CL-nanoparticle will continue to be exploited for an increasingly diverse range of analytical determinations, especially in biomedical applications and immunoassay.

\section{Acknowledgement}

The authors would like to acknowledge the Research Centre, College of Science, King Saud University, Riyadh, Saudi Arabia, for support.

\section{References}

Alves C M, Marzocchi-Machado C M, Carvalho I F and Lucisano Valim Y M 2003 Talanta 60601

Cui H and Dong Y P 2006 J. Electroanal. Chem. 59537

Cui H, Zhang Z F and Shi M J 2005 J. Phys. Chem. B109 3031

Chen H, Gao F, He R and Cui D 2007 J. Colloid Interf. Sci. 315158

Chen X, Wang C, Tan X and Wang J 2011 Anal. Chim. Acta 68992

Du J X, Li Y H and Guan R 2007 Microchim Acta 158145

Guo J Z and Cui H 2007 J. Phys. Chem. C111 12254

Gorman B A, Francis P S, Dunstan D E and Barnett N W 2007 Chem. Commun. 4395

Lee J M, Karim M M and Lee S H 2005 J. Fluoresc. 15735

Lee H S, Karim M M and Alam S M 2007 Luminescence 22331

Li M and Lee S H 2005 Microchim. J. 80237

Li S, Li X Z, Xu J and Wei X W 2008 Talanta 7532

Li S, Zhang X M, Yao Z J, Yu R, Huang F and Wei X W 2009a J. Phys. Chem. C113 15586

Li S, Zhang X M, Du W X, Ni Y H and Wei X W 2009b J. Phys. Chem. C113 1046

Li S, Li X Z, Zhang Y Q, Huang F, Wang F F and Wei X W 2009c Microchim Acta 167103

Li Y, Niu W and Lua J 2007 Talanta 711124

Liu B, He Y, Duan C, Li N and Cui H 2011 J. Photochem. Photobiol. A: Chem. 21762

Niazov T, Pavlov V, Xiao Y, Gill R and Willner I 2004 Nano Lett. 4 1683

Sau T K, Pal A and Pal T 2001 J. Phys. Chem. B105 9266 
Su Y, Wang J and Chen G 2005 Talanta 65531

Tsukada S, Miki H, Lin J M, Suzuki T and Yamada M 1998 Anal. Chim. Acta 371163

Waseem A 2007 Int. J. Environ. Anal. Chem. 87825

Xie X, Shao X, Yue Q, Huang C and Song Z 2007 Anal. Lett. 40 1951
Xu L S and Cui H 2007 Luminescence 2277

Zhang Z F, Cui H, Lai C Z and Liu L Z 2005 Anal. Chem. 77 3324

Zhang Z F, Cui H and Shi M J 2006 Phys. Chem. 81017

Zhang Z Y, Zhang C and Zhang X R 2002 Analyst 127792

Zhao S, Bai W, Wang B and He M 2007 Talanta 73142 